\title{
Crystal Orientation and Grain Size: Do They Determine Optoelectronic Properties of $\mathrm{MAPbl}_{3}$ Perovskite?
}

\author{
Loreta A. Muscarella, ${ }^{\dagger}$ Eline M. Hutter, ${ }^{\dagger}$ Sandy Sanchez, ${ }^{\dagger}$ Christian D. Dieleman, \\ Tom J. Savenije, ${ }^{\S \odot}$ Anders Hagfeldt, ${ }^{\ddagger}$ Michael Saliba, ${ }^{\|, \perp}$ and Bruno Ehrler ${ }^{* \dagger \odot}$ \\ Center for Nanophotonics, AMOLF, Science Park 104, 1098 XG Amsterdam, The Netherlands \\ ${ }^{\ddagger}$ Laboratory of Photomolecular Science (LSPM), École Polytechnique Fédérale de Lausanne (EPFL), Station 6, 1015 Lausanne, \\ Switzerland \\ ${ }^{\S}$ Department of Chemical Engineering, Delft University of Technology, Van der Maasweg 9, 2629 HZ Delft, The Netherlands \\ "Institute of Materials Science Technical, University of Darmstadt, Alarich-Weiss-Strasse 2, D-64287 Darmstadt, Germany \\ ${ }^{\perp}$ IEK-5 Photovoltaik, Forschungszentrum Jülich GmbH, 52425 Jülich, Germany
}

\section{Supporting Information}

\begin{abstract}
Growing large, oriented grains of perovskite often leads to efficient devices, but it is unclear if properties of the grains are responsible for the efficiency. Domains observed in SEM are commonly misidentified with crystallographic grains, but SEM images do not provide diffraction information. We study methylammoinium lead iodide $\left(\mathrm{MAPbI}_{3}\right)$ films fabricated via flash infrared annealing (FIRA) and the conventional antisolvent (AS) method by measuring grain size and orientation using electron backscattered diffraction (EBSD) and studying how these affect optoelectronic properties such as local photoluminescence
\end{abstract}
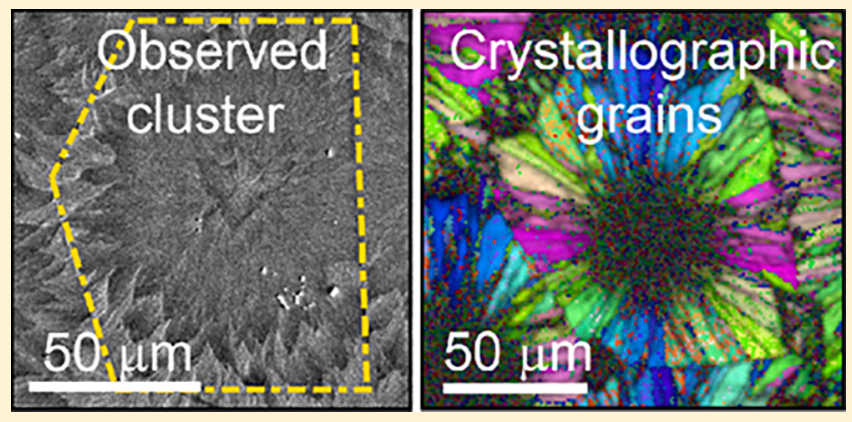
(PL), charge carrier lifetimes, and mobilities. We observe a local enhancement and shift of the PL emission at different regions of the FIRA clusters, but we observe no effect of crystal orientation on the optoelectronic properties. Additionally, despite substantial differences in grain size between the two systems, we find similar optoelectronic properties. These findings show that optoelectronic quality is not necessarily related to the orientation and size of crystalline domains.

$\mathrm{H}$ ybrid perovskites have recently gathered significant attention due to the high efficiency of perovskite-based solar cells and other optoelectronic devices. ${ }^{1,2}$ One of the most surprising properties of these materials is that the performance is very tolerant to different methods of fabrication, ${ }^{3-5}$ different compositions, ${ }^{6,7}$ and different chemical treatments. ${ }^{8-10}$ This is reflected in high photoluminescence quantum efficiencies (PLQEs), ${ }^{11,12}$ which are a measure of the fraction of radiative versus nonradiative decay and therefore a direct measure of the optical quality. In solar cells, the PLQE, for example, is directly related to the open-circuit voltage. ${ }^{13}$ In practice, the PLQE is reduced by the presence of defects, ${ }^{13,14}$ which are often related to chemical impurities such as interstitials, vacancies, dangling bonds, ${ }^{15,16}$ or defects on the surface and grain boundaries (GBs). ${ }^{17-19}$ Both bulk and surface defects have been extensively studied in perovskites, ${ }^{20-23}$ and efficient passivation strategies are now routinely employed to achieve high light emitting diode (LED) and solar cell efficiencies. ${ }^{24-26}$ In addition, chemical methods (e.g., Lewis bases ${ }^{21}$ or chloridebased additives ${ }^{6,27}$ ) in the perovskite precursor are often applied to grow larger grains, which has been thought to suppress nonradiative recombination pathways by reducing the number of GBs. ${ }^{28-30}$ Furthermore, these changes in the synthesis route affect the crystal growth and therefore the preferred crystallographic orientations. ${ }^{8,31,32}$

However, it is unclear if the changes in grain size and orientation obtained by these treatments cause the improved optoelectronic properties or if these are mainly related to passivation effects from the additives. Crystallographic orientation and their relation to the PL and other properties have not been studied in thin films so far because spatial resolution of the crystallographic parameters was lacking. Furthermore, the morphological "grain" observed in SEM images does not necessarily correspond to a crystallographic grain; therefore, additional information is needed in order to relate the grain size to optoelectronic properties.

Here we use EBSD to measure size, orientation, and rotation of crystallographic grains in polycrystalline $\mathrm{MAPbI}_{3}$ films with high spatial resolution. We study a $\mathrm{MAPbI}_{3}$ thin film where crystallization is induced by FIRA, a low-cost and rapid

Received: September 18, 2019

Accepted: September 23, 2019

Published: September 23, 2019 
a)

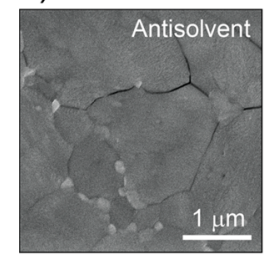

b)

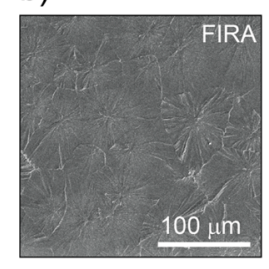

c)

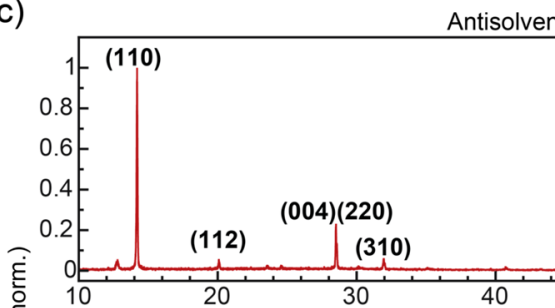

$2 \theta$

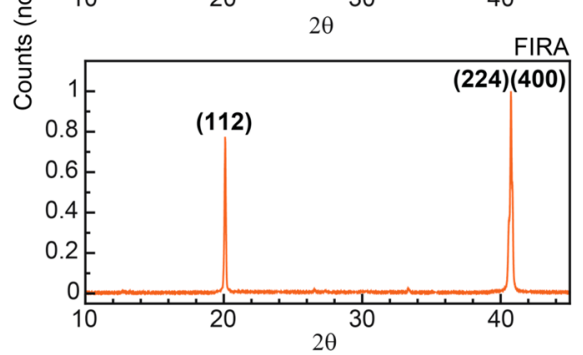

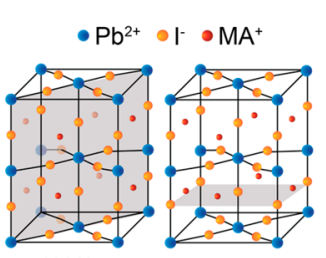

(110)

(004)

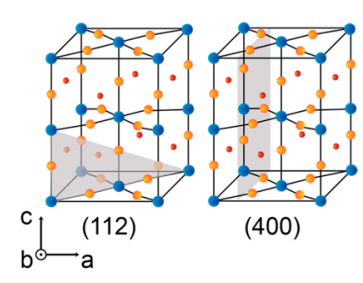

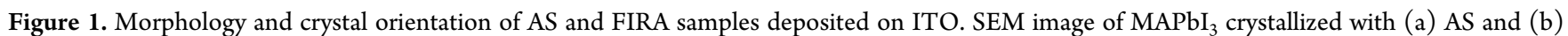
FIRA; (c) XRD pattern of AS and FIRA MAPbI 3 with the corresponding unit cell cut along the (110) and (004) planes for the AS sample and along (112) and (400) for the FIRA sample.

synthesis method. ${ }^{33,34}$ These films exhibit large grains (tens of micrometers) highly oriented along the (112) and (400) planes in the direction parallel to the substrate. We find that the growth is spherulitic, i.e., needle-like arrays, yielding $\sim 100$ $\mu \mathrm{m}$ sized clusters that consist of radially grown grains. With EBSD mapping, we find that the two crystal orientations in the FIRA films are well-separated in pairs in the large clusters of grains. We compare the PL from these clusters and find that the PL intensity and spectrum are the same for the two crystal orientations. These results suggest that the crystallographic orientation does not govern the optoelectronic quality of perovskite thin films. Similarly, we find comparable emission in the interior part of the grain and at the GBs, but we find enhanced emission and a red shift at the cluster boundaries (CBs) and at the nucleation sites, which we attribute to favorable light-outcoupling and self-absorption. Finally, we compare the FIRA sample to one where crystallization is induced by the conventional AS dripping method, from the same precursor solution. This method produces submicron grains with random orientation. In our case, both samples show a comparable charge carrier mobility and lifetime, demonstrating that these properties are not necessarily determined by the grain size, at least for grains above a few hundred nanometers.

To study the relation between the perovskite crystal orientation and its optoelectronic properties we first synthesize $\mathrm{MAPbI}_{3}$ on Indium Tin Oxide (ITO) via FIRA wherein the spin coated perovskite film is annealed using a short $(1.2 \mathrm{~s})$, highly intense infrared illumination to induce nucleation, as previously reported. ${ }^{33}$ For comparison we also fabricate a sample by the AS method where chlorobenzene is rapidly poured onto the liquid precursor while spin coating. Both the FIRA and AS sample were fabricated from the same precursors under identical conditions, and thus the only difference is the crystallization process.

We probe the morphology of both samples using scanning electron microscopy (SEM), as shown in Figures la,b and S1. SEM images show a dramatic difference in cluster size from the AS method $(100 \mathrm{~nm}-2 \mu \mathrm{m})$ compared to FIRA $(\sim 100 \mu \mathrm{m})$. The differentiation between morphological clusters observed in the SEM and real crystallographic grains is still largely misunderstood, ${ }^{35,36}$ and often, these clusters seen in SEM images are assigned to grains. In crystallography, the term "grain" is defined by a coherently diffracting domain of solidstate matter that has the same structure as a single crystal. ${ }^{37}$ Therefore, from SEM images alone, it is not possible to define the apparent domains as crystallographic grains because diffraction information is not measured. For this reason, we use "clusters" to describe the large perovskite domains shown in SEM images.

In addition, we analyze the bulk crystal structure of the two systems deposited on ITO using X-ray diffraction (XRD). The AS sample shows a tetragonal XRD pattern where peaks from (110), (112), (220), and (310) planes arise from the background. ${ }^{38,39}$ In contrast, the FIRA sample shows a strong preferential orientation along the (112) and (400) planes (Figure 1c). A cutoff of the primitive tetragonal cell and the planes that show the highest diffraction peaks are shown next to the diffraction patterns.

To investigate the crystal growth of the FIRA sample, we apply infrared annealing with varying pulse duration (Figure $2 \mathrm{a})$. After $0.2 \mathrm{~s}$ of annealing, we observe a needle-like crystal morphology. After $0.5 \mathrm{~s}$, the crystals have grown in a spacefilling manner by branching from the parental needle until each domain impinges with neighboring clusters, resulting in straight boundaries. Optical microscopy of the final films (Figure 2b) shows large perovskite clusters in agreement with the SEM images. We use polarized light microscopy to find how the two orientations observed in the XRD are spatially distributed. Figure $2 \mathrm{~b}$ shows the presence of paired oriented domains within FIRA films for all of the clusters (see also Figure S2), originating from the change in polarization of the incident polarized light caused by the two different refractive indices in the different crystal directions. This is a indication of spherulitic growth (schematically shown in Figure 2c) via noncrystallographic branching ${ }^{40}$ typical for many polymeric materials ${ }^{41}$ and inorganic salts. ${ }^{42}$ Interestingly, there also seems to be a common direction to all clusters, suggesting a global effect from the temperature or strain gradient. In general, spherulitic growth requires the use of a saturated solution, high viscosity, and slow crystallization. This growth is also catalyzed by the presence of impurities ${ }^{40}$ or strain in the material. In this 
a)

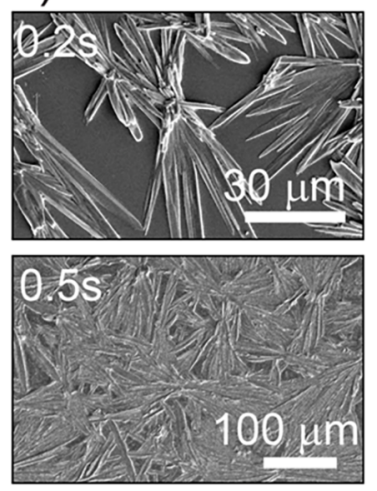

b)

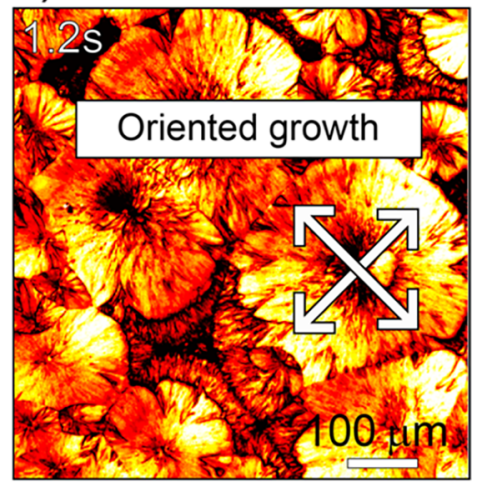

c)

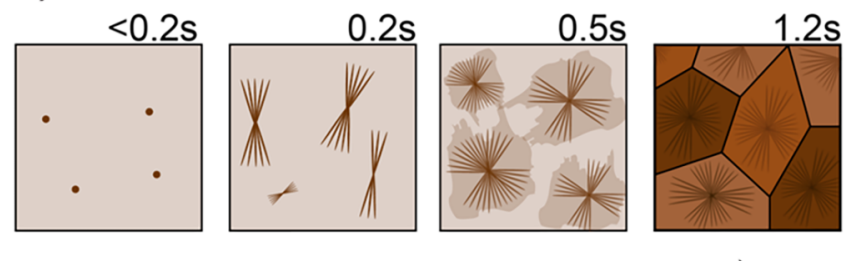

FIRA annealing time (s)

Figure 2. Spherulitic growth mechanism of $\mathrm{MAPbI}_{3}$ results in paired oriented domains. (a) SEM of the FIRA film after 0.2 and $0.5 \mathrm{~s}$ of infrared annealing. (b) Polarized microscopy image of the final FIRA film showing paired oriented perovskite domains. (c) Schematic spherulitic growth of perovskite films after $0.2,0.5,1$, and $1.2 \mathrm{~s}$ of FIRA annealing

case, $\mathrm{MAPbI}_{3}$ and the ITO (or quartz) show a substantial difference in the thermal expansion coefficient $\left(\alpha_{\mathrm{MAPbI} 3}=6.1 \times\right.$ $10^{-5} \mathrm{~K}^{-1}, \alpha_{\text {ITO }}=0.85 \times 10^{-5} \mathrm{~K}^{-1}, \alpha_{\text {quartz }}=0.37 \times 10^{-5} \mathrm{~K}^{-143}$ ), which has been shown to be the origin of strain during the cooling process after the thermal annealing. ${ }^{44}$ Thus, strain can be considered as a factor inducing spherulitic growth in our system.

We study the crystal grains and their orientation with high spatial resolution $(10 \mathrm{~nm})$ EBSD, which is commonly used for investigations of grains in metal alloys, ${ }^{45}$ strain, ${ }^{46}$ and the nature of GBs. ${ }^{47}$ EBSD is a SEM-based technique where the incident electron beam interacts with a crystalline material and electron back-scattered patterns (Kikuchi patterns) are produced by incoherent wide-angle electron scattering (thermal diffuse scattering) from the specimen. A scheme of the setup is depicted in Figure 3a. For a full description of EBSD measurements, see the Supporting Information section S1.

The main limitation that has restricted its application for perovskites is related to the high electron current needed for the phosphor screen to collect a sufficiently large signal-tonoise ratio. In the case of perovskites containing organic cations, the use of a current of a few $\mathrm{nA}$ can already be damaging to the material. Recently, Adhyaksa et al. ${ }^{48}$ pioneered the application of EBSD for $\mathrm{MAPbBr}_{3}$ using a direct electron detector, which allows for a low accelerating voltage of $5 \mathrm{kV}$ instead of $30 \mathrm{kV}$ and low sample currents of $\mathrm{pA}$ instead of $\mathrm{nA}$ in conventional systems. We used the same detection system to collect the Kikuchi patterns from our $\mathrm{MAPbI}_{3}$ films. The obtained Kikuchi patterns allow for the identification of grains, their size and shape, and the nature of boundaries between them. By fitting the patterns, we can identify the crystal phase, grain orientation, and rotation, as described in Supporting Information section S1.

Importantly, because with EBSD diffraction information is measured, we can distinguish clusters from grains and define the crystallographic grain size of $\mathrm{MAPbI}_{3}$ fabricated via FIRA and AS. Furthermore, being a spatially resolved technique, it allows for direct comparison of a specific crystallographic orientation or a specific grain size with other spatially resolved optoelectronic techniques. Figure $3 b, c$ shows an overlay of the image quality (brightness, IQ) with the inverse pole figure (color, IPF) of the AS and FIRA system along the $z$-axis (normal to the substrate). The IQ maps the sharpness of the Kikuchi lines obtained from the EBSD measurement, which gives qualitative indications about the crystallinity of the material, topographic effects (e.g., roughness), strain of the microstructure, and GBs (for more details, see Supporting Information section S1). The IPF represents the crystal orientation obtained from fitting the Kikuchi patterns to each pixel of the image with respect to a reference axis. Along the $z$-axis, the AS sample (Figure $3 b$ ) shows randomly oriented grains of hundreds of nanometers. On the contrary, in the FIRA sample (Figure 3c), all grains are aligned along [100] and [112] directions (green and purple colors), and the two orientations are paired in larger regions, in agreement with $\mathrm{XRD}$ and polarized microscopy measurements. The distribution of orientations for the two samples clearly shows the mostly random orientation for the AS sample and the bipolar distribution of orientations for the FIRA sample (Figure 3d). Consistent with XRD (Figure 1c), we observe significant orientation along both the [100] and [112] directions, but locally, the ratio can vary (see Figure S3). Orientation maps along $x$ - and $y$-directions (parallel to the substrate) show random grain orientations for the AS sample (see Figure S4). On the other hand, for the FIRA sample, they show highly oriented and paired grains (Figures $3 \mathrm{e}, \mathrm{f}$ and S5 for orientation distribution). Here, GBs within the FIRA cluster and the nucleation site are shown in dark gray (low IQ value) because they are largely smaller than the step size (see Figure S3 for a cluster center with higher resolution). A grain, by definition, has the same orientation in all three directions. More than $90 \%$ of the grains that we measure are smaller than $1 \mu \mathrm{m}^{2}$ for the AS sample, while the FIRA samples show $83 \%$ of the grains having an area between 0 and $30 \mu \mathrm{m}^{2}$ (see Figure S6). While the FIRA grains are large, they are smaller than the size of the clusters shown in SEM (Figure 3g).

$\mathrm{MAPbI}_{3}$ has an anisotropic tetragonal crystal structure, and properties like trap-state density have been shown to be dependent upon crystal orientation. ${ }^{49,50}$ Here, we study the optoelectronic properties of the two well-characterized and spatially separated orientations shown by the FIRA sample using spatially resolved PL. Often, enlargement and preferential orientation of grains are achieved by introducing chemical additives that often also passivate the perovskite grains, prolonging the lifetime and enhancing the PL. To avoid these effects, we fabricate a highly oriented FIRA sample without additives from the same precursor solutions as the AS sample.

We measure the PL intensity using a confocal imaging microscope using a $405 \mathrm{~nm}$ laser as the excitation source with a power density of $0.23 \mathrm{~W} / \mathrm{cm}^{2}$. As the two orientations on the sample are spatially well-separated, we can map any difference in PL emission between them. We measure a large area of the 
a)

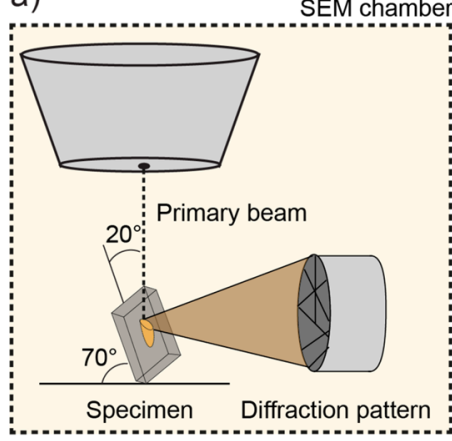

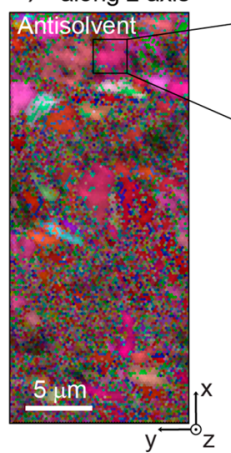

c)

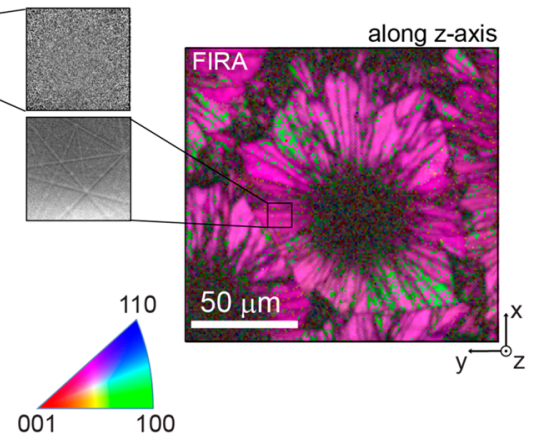

d)
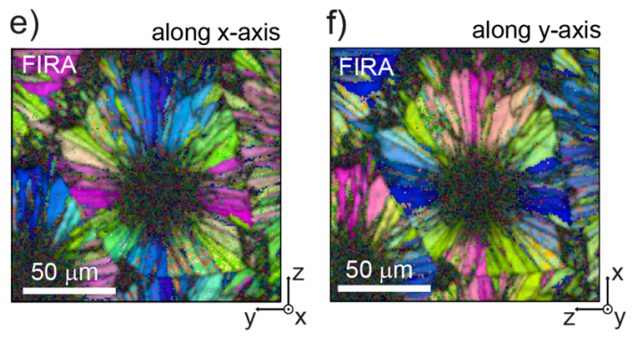

g)
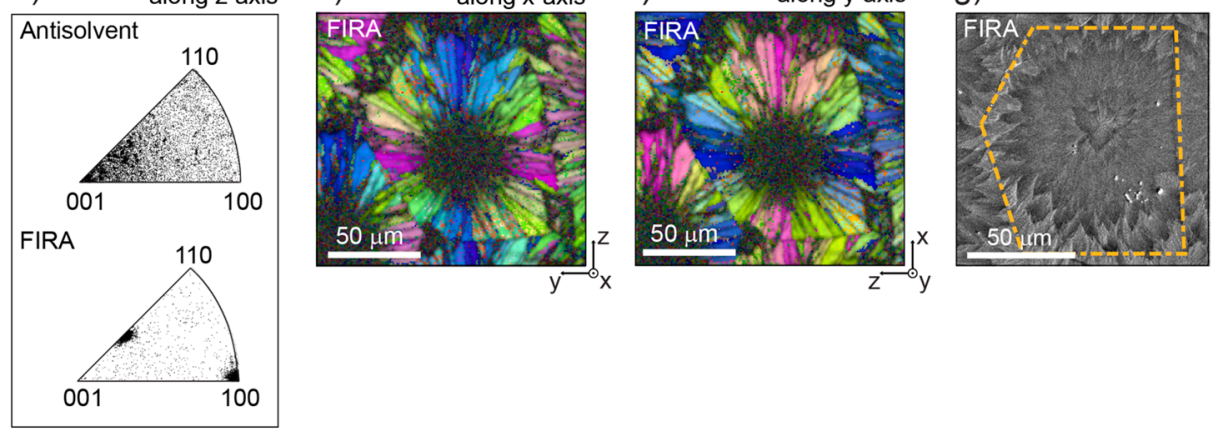

Figure 3. EBSD maps reveal the crystal orientation and grain size of the two systems. (a) EBSD setup. (b) IQ (brightness) overlay with the IPF map of the AS sample showing the crystallographic orientation along the $z$-direction. (Inset) Magnification of the typical Kikuchi patterns recorded from the sample. (c) IQ (brightness) overlay with the IPF map of the FIRA sample showing the crystallographic orientation along the $z$-direction. (Inset) Magnification of the typical Kikuchi patterns recorded from the sample. (d) Distribution of orientation for the two samples along the $z$ direction. (e) IQ (brightness) overlay with the IPF map of the FIRA sample showing the crystallographic orientation along the $x$-direction and (f) $y$-direction. (g) SEM image showing the apparent grain size of a FIRA cluster. The cluster measured with EBSD is highlighted with a dashed line.

FIRA and AS samples including a whole FIRA cluster (Figures $4 \mathrm{a}$ and S7 for a larger area). In the AS sample, the PL intensity is relatively homogeneously distributed across the measured region, varying from cluster to cluster, consistent with many other works. ${ }^{51,52}$ For the FIRA sample, we find comparable PL between the interior of the grain and the GBs. Previously, GBs were reported to cause either enhancement or reduction ${ }^{29,48,53}$ of the emission intensity, mostly caused by ambiguities of assigning these boundaries. In our case, GBs are not detrimental for the optoelectronic properties of the film. In contrast, the FIRA sample shows an enhancement in intensity of 2- to 6-fold at the CBs and at the nucleation site where the spherulitic growth is initiated.

The map of the PL peak position of the AS sample (Figure 4b) shows an identical emission wavelength for every cluster. On the contrary, the FIRA sample shows a variation in emission wavelengths at different locations. The PL peak position at the $\mathrm{CBs}$ and nucleation point is red-shifted compared to the interior of the cluster (Figure 4b). We also observe an asymmetric shape of the peak for the FIRA sample (Figure S8). This shape in combination with the red shift of the peak has been assigned to self-absorption when light travels through the perovskite layers before being emitted. ${ }^{54}$ To validate this assignment, we calculate the emission spectra expected from the light passing through different thicknesses of $\mathrm{MAPbI}_{3}$ before being emitted. We observe the red shift at the $\mathrm{CBs}$ and nucleation points corresponding to light that has been traveling through $400-800 \mathrm{~nm}$ excess material as compared to the cluster interior region before being emitted (additional traveled thickness (ATT) on the horizontal scale bar in Figure $4 \mathrm{~b}$; see Supporting Information section S2 for calculation details); this is in good agreement with the observed thickness variation between the two regions in atomic force miscroscopy (AFM). We see no difference in wavelength or intensity for the regions that correspond to the two different, well-defined crystal orientations. Hence, the variations in $\mathrm{PL}$ emission intensity and wavelength are not related to the crystallographic orientation.

From AFM measurements, the AS sample shows only minor height variation between the center and the rest of the grain (RMS roughness $70.5 \pm 7.5 \mathrm{~nm}$, Figure $4 \mathrm{c}$ ). On the contrary, the FIRA sample shows significant height variation at the CBs and at the nucleation point (Figures $4 \mathrm{c}$ and S9). In these regions, the film is around $400-1000 \mathrm{~nm}$ thicker compared to the interior of the cluster, leading to a much larger roughness (the RMS roughness is estimated to be $26.8 \pm 14.5 \mathrm{~nm}$ in the interior of the cluster, $175 \pm 47 \mathrm{~nm}$ at the nucleation site, and $75.3 \pm 21 \mathrm{~nm}$ at the CBs). The rough nature of the boundary can favor light-outcoupling, as shown on patterned perovskite surfaces. ${ }^{55}$ We hence tentatively assign the higher PL intensity in the CBs and nucleation site to better light-outcoupling at these rough surfaces.

We corroborate the assignment of self-absorption by measuring PL lifetime maps of the FIRA sample. We observe a biexponential decay, with a fast and a slow component. The effect of reabsorption and photon recycling have been shown 
a)

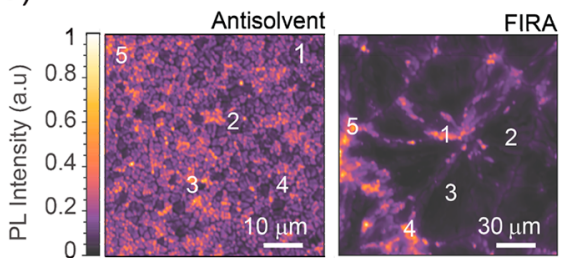

c)

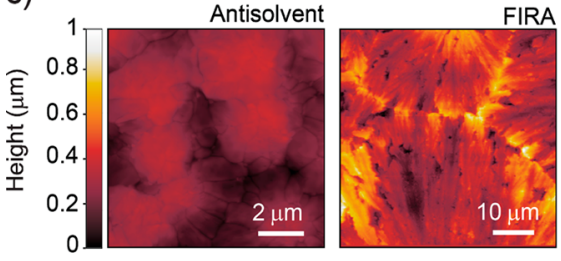

b)

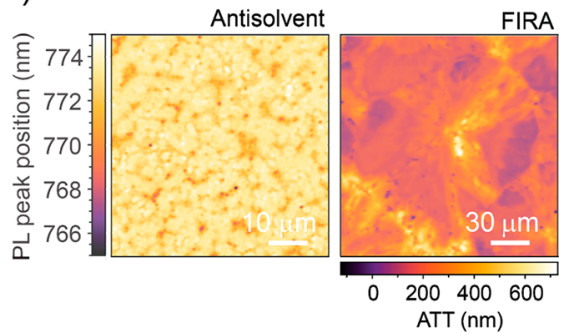

d)

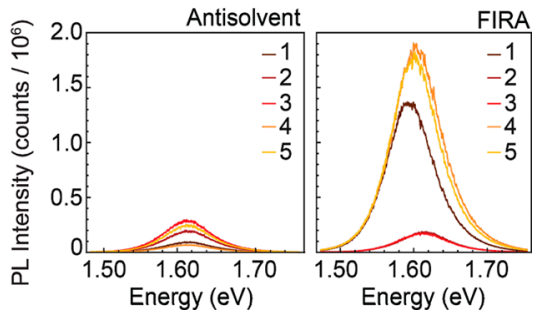

Figure 4. Emission properties of $\mathrm{MAPbI}_{3}$ varying crystal orientation and grain size. (a) Spatially resolved PL of the AS and FIRA samples. (b) Emission wavelength map of the same region as in figure (a) for the AS and FIRA samples. For the FIRA sample, the emission wavelength is converted into the ATT (on the horizontal scale bar) the light has been through before it is emitted. (c) AFM image of the AS and FIRA samples, highlighting the CB region in the FIRA sample. (d) PL spectra extracted from five random regions indicated in panel (a) in the AS sample showing similar PL intensity and no shift in the peak position and PL spectra extracted from the CBs and the inner cluster region of the FIRA sample, showing enhancement in the PL at the CBs and a red shift of the peak due to self-absorption.

to affect the recombination dynamics by lengthening the slow lifetime component. ${ }^{55-58}$ The lifetime of the FIRA sample at the CBs and at the nucleation site shows the same fast lifetime component but a prolonged slow decay component compared to the interior of the cluster (e.g., $\tau_{1}=10.1 \pm 0.9$ and $\tau_{2}=49.2$ \pm 2.0 for the darker PL region in the grain interior and $\tau_{1}=$ $12.9 \pm 0.9$ and $\tau_{2}=75.1 \pm 1.1$ for the brighter PL region at the $\mathrm{CBs}$; see Figure S10). The ratio of the slow lifetime component (1.5 in our case) depends on the thickness and light-outcoupling events. ${ }^{59}$ The increased thickness increases the likelihood of reabsorption and increases the ratio, and better light-outcoupling decreases the lifetime ratio. Accounting for only the difference in thickness at the CBs compared to the cluster interior, the lifetime ratio is overestimated. However, outcoupling is also most likely at rougher regions such as the CBs. Thus, the change in lifetime can be explained by taking both the larger thickness and outcoupling into account, consistent with the shift in the PL spectrum (see Supporting Information section S3 for semiquantitative analysis).

Similarly to the PL intensity, no significant difference in lifetime is observed between the interior of the grain and the GBs within the FIRA cluster. We also do not observe a trend across the cluster that would correspond to the two different grain orientations. Thus, there is no direct correlation between the crystal orientation and the PL intensity.

To investigate the role of grain size on the PL intensity, we compare the PL from the interior of the FIRA sample to the AS sample. In Figure 4d, we plot PL spectra from five random regions of the two samples. Here we show that the interior region of the FIRA cluster shows a comparable PL intensity as the AS (FIRA points 2 and 3), despite the much larger size of the FIRA grains. This comparison shows that the PL emission is not determined by solely the grain size (at least for grains > $400 \mathrm{~nm})$.

Next to the optical properties, the electronic properties have been shown to depend on grain size in some cases. ${ }^{60}$ To investigate the mobility and recombination dynamics of photoexcited charge carriers in our two systems, we used the time-resolved microwave conductivity (TRMC) technique. The FIRA and AS samples were excited with a $485 \mathrm{~nm}$ excitation wavelength. Figure $5 \mathrm{a}, \mathrm{b}$ shows the photoconduc-

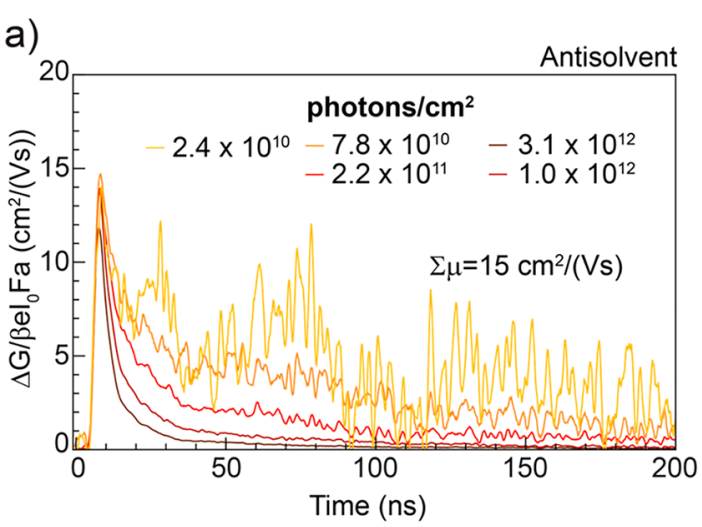

b)

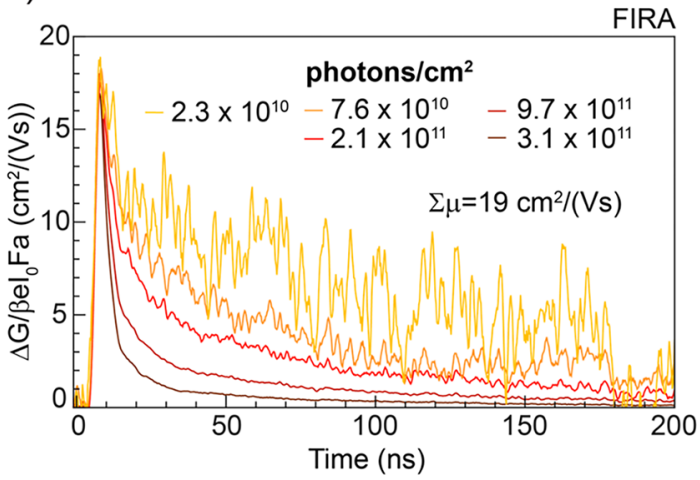

Figure 5. Mobility and lifetime varying the grain size. TRMC traces measured at different carrier densities for the (a) AS and (b) FIRA samples deposited on quartz. 
tance $\Delta G$ as a function of time after pulsed excitation of AS and FIRA samples, respectively. The product of the yield of free charges $\varphi$ and their mobility $\sum \mu$ (sum of electron and hole mobility) was derived from the maximum signal height $\left(\Delta G_{\max }\right)$, which was divided by the fraction of absorbed photons for the two samples to take in account the difference in absorption. We found a mobility of $(15 \pm 3) \mathrm{cm}^{2} /(\mathrm{V} \mathrm{s})$ for the AS and $(19 \pm 4) \mathrm{cm}^{2} /(\mathrm{V} \mathrm{s})$ for the FIRA sample, which is comparable to sample-to-sample variation. The charge carrier lifetime was obtained from the photoconductance decay. The decay of the photoconductance represents the immobilization of free charges due to trapping or recombination. For both systems, we found that the lifetime of charges is on the order of a few hundred nanoseconds. We observed a slight increment of the effective mobility in the FIRA sample compared to the AS sample, likely related to the enlarged grain size. ${ }^{60}$ This difference is relatively small, despite the difference in grain size between the FIRA (tens of microns) and the AS sample (hundreds of nanometers). This shows that the grain size only does not dominate charge carrier transport properties. We note that the TRMC measurement mostly probes the local conductivity $(\sim 50 \mathrm{~nm}$, more details about the probing length are reported in Supporting Information section S4). Intergrain transport across larger distances may show larger differences in crystallographically different systems. This finding is consistent with the similar device performances that have been reported for both FIRA and AS, showing similar $J_{\text {sc }} V_{\text {oc }}$ FF, and PCE. ${ }^{33}$ Practically, FIRA could allow a lower-cost, environmentally friendly fabrication route to produce large scale, reproducible, and more stable (see Figure S11) perovskites compared to the AS method. ${ }^{33}$

In conclusion, we have shown that the crystallographic orientation of $\mathrm{MAPbI}_{3}$ grains does not determine the optical and local electronic properties. We studied a $\mathrm{MAPbI}_{3}$ thin film where crystallization is induced by FIRA. We applied EBSD to extract information about the microstructure of the perovskite thin film with high spatial resolution. The large FIRA clusters consist of grains that are tens of micrometers in size, highly oriented perpendicularly and in parallel to the substrate with a preferential orientation along the [112] and [100] directions along the $z$-axis. In comparison, a conventional sample fabricated via AS shows randomly oriented grains of hundreds of nanometers in the three directions. We found increased PL intensity and a spectral shift in the FIRA sample at the CBs compared to the interior part of the FIRA cluster, which can be explained by roughness and thickness variations favoring lightoutcoupling and self-absorption, respectively. No difference in PL between the grain and the GBs was observed in the FIRA sample, leading us to the conclusion that the GBs are not detrimental to the PL in our system. Finally, we investigated charge carrier dynamics and found a comparable lifetime and a slight increment in effective mobility in the FIRA and AS samples. We hence conclude that in our case neither the grain size (when larger than a few hundreds of $\mathrm{nm}$ ) nor the grain orientation are the dominant factor determining the optoelectronic properties of perovskite thin films. This finding implies that efforts toward a more efficient perovskite device may need to focus on reducing defects within the bulk and at the interface as well as impurities within the materials rather than growing large, oriented grains.

Methods. The fabrication of the two systems (AS dripping and FIRA method) is reported elsewhere. ${ }^{33}$
The XRD pattern of perovskite films deposited on ITO was measured using an X-ray diffractometer, Bruker D2 Phaser, with $\mathrm{Cu} \mathrm{K} \alpha 1.5406 \AA$ as the X-ray source, $0.002^{\circ}(2 \theta)$ as the step size, and $0.150 \mathrm{~s}$ as the exposure time. An FEI Verios 460 instrument was used to obtain SEM images. AFM measurements were performed on a Veeco Dimension 3100 (Bruker) in tapping mode.

An optical microscope (Zeiss, AxioCam ICc 5) equipped with a $10 \times / 0.2$ objective EC Epiplan, polarizer, and analyzer set at different angles was used for the polarized optical microscopy image. We combined the optical microscope in reflection mode with two polarizers, one placed in the light path before the specimen and a second one, called the analyzer, between the objective lenses and eyepieces.

Steady-state PL of samples deposited on quartz was measured with a home-built setup equipped with a $640 \mathrm{~nm}$ continuous-wave laser as the source of excitation (PicoQuant LDH-D-C-640) at a power output of $1 \mathrm{~mW}$. Two Thorlabs filters, a long-pass, ET655LP, and a notch, ZET642NF, were used to remove the excitation laser from the signal. The PL was coupled into a fiber connected to an OceanOptics USB4000 spectrometer. An integration time of $300 \mathrm{~ms}$ was used for each measurement.

For EBSD measurements, samples were deposited on ITO to avoid charging effects during the experiment. The detector used was a direct electron detector based on the Timepix sensor from Amsterdam Scientific Instruments (ASI). The best parameters for the scans were found to be $15 \mathrm{keV}$ as the voltage, $100 \mathrm{pA}$ as the current, $100 \mathrm{~ms}$ as the exposure time, and working distances between 12 and $10.1 \mathrm{~mm}$. This translates to the application of $10 \mathrm{nA} \mathrm{ms}$ electron dose per pixel, which is around $10^{3}$ times lower compared conventional measurement, reducing sample damage. The step size was chosen depending on the cluster size shown by the specific sample, $200 \mathrm{~nm}$ and $1 \mu \mathrm{m}$ for the AS and FIRA samples, respectively. EBSD data were collected using EDAX OIM software, and a Python script was used for image processing. The resulting Kikuchi patterns were indexed using tetragonal symmetry, $I 4 / \mathrm{mcm}$, using $1-3^{\circ}$ as the degree of tolerance. A detailed procedure for fitting the Hough's space is reported in Supporting Information section S1.

A spatially resolved PL map was measured using a confocal imaging microscope (WITec alpha300 SR). A $405 \mathrm{~nm}$ laser diode (Thorlabs S1FC405) was used as the excitation source, where the PL intensity of the two $\mathrm{MAPbI}_{3}$ films was collected in reflection mode through a NA 0.9 objective using a spectrometer (UHTC 300 VIS, WITec), leading to a spatial resolution of $0.33 \mu \mathrm{m}$. The intensity was measured within the $700-840 \mathrm{~nm}$ emission wavelength range. A $488 \mathrm{~nm}$ LP filter was used to remove the excitation laser from the signal. The light collection was done from the same sample side as the excitation. The PL spectra were converted to the energy scale using a Jacobian transformation. ${ }^{61}$

TRMC was measured on AS and FIRA samples deposited on quartz. The thin films were placed in a sealed resonance cavity inside of a nitrogen-filled glovebox to avoid degradation due to air exposure. The samples were excited at $485 \mathrm{~nm}$ using a pulsed excitation $(10 \mathrm{~Hz})$ and the photoconductance probed at different excitation densities. Neutral density filters were used to vary the intensity of the incident light. The $\Delta G$ signal rise was limited by the width of the laser pulse ( $3.5 \mathrm{~ns}$ fwhm) and the response time of the microwave system (18 ns). The slow repetition rate of the laser of $10 \mathrm{~Hz}$ ensures full relaxation 
of all photoinduced charges to the ground state before the next laser pulse hits the sample. The mobility is derived by the maximum signal height as the following equation ${ }^{62}$

$$
\varphi \sum \mu=\frac{\Delta G_{\max }}{\beta e I_{0} F_{\mathrm{A}}}
$$

where $I_{0}$ is the number of photons per unit area per pulse, $\beta$ is a geometric factor related to the microwave cell, $e$ is the elementary charge, and $F_{\mathrm{A}}$ the fraction of light absorbed by the sample at the excitation wavelength used. We assume $\varphi$ to be unitary for the low exciton binding energy of the material.

Time-correlated single-photon counting (TCSPC) measurements were performed with a home-built setup equipped with PicoQuant PDL 828 "Sepia II" and a PicoQuant HydraHarp 400 multichannel picosecond event timer and TCSPC module. A $640 \mathrm{~nm}$ pulsed laser (PicoQuant LDH-D-C-640) with a repetition rate of $2 \mathrm{MHz}$ was used to excite the sample. A Thorlabs FEL-700 long-pass filter was used to remove the excitation laser.

\section{ASSOCIATED CONTENT}

\section{S Supporting Information}

The Supporting Information is available free of charge on the ACS Publications website at DOI: 10.1021/acs.jpclett.9b02757.

SEM at lower magnification of the AS sample; polarized optical microscopy images of FIRA with the polarizer and analyzer at different angles; EBSD measurement of a FIRA cluster; crystal orientation distribution along $x$ and $y$-directions for AS and FIRA samples; IQ and IPF overlay showing the orientation along the $x$ - and $y$ directions for the AS sample; grain size distribution for AS and FIRA obtained from EBSD measurement; spatially resolved PL of a bigger area of the FIRA sample; AFM of a different $\mathrm{CB}$ region in the FIRA sample; lifetime map of the FIRA sample at the CB region; normalized PL spectra of five random regions in the AS and FIRA samples; EBSD geometry, Hough transformation, and pattern indexing; and additional thickness traveled by light as a function of the emission wavelength in $\mathrm{MAPbI}_{3}$ fabricated by FIRA (PDF)

\section{AUTHOR INFORMATION}

\section{Corresponding Author}

*E-mail: b.ehrler@amolf.nl.

\section{ORCID $\odot$}

Loreta A. Muscarella: 0000-0002-0559-4085

Eline M. Hutter: 0000-0002-5537-6545

Sandy Sanchez: 0000-0003-0167-5790

Tom J. Savenije: 0000-0003-1435-9885

Michael Saliba: 0000-0002-6818-9781

Bruno Ehrler: 0000-0002-5307-3241

\section{Notes}

The authors declare no competing financial interest.

\section{ACKNOWLEDGMENTS}

The authors thank Erik C. Garnett for providing feedback on the paper and Andries Lof for technical support during the EBSD measurements. This work is part of the research program of The Netherlands Organization for Scientific Research (NWO). S.S. and M.S. thank the Adolphe Merkle
Institute at the University of Fribourg, Switzerland, for past support.

\section{REFERENCES}

(1) Chen, Y.; Zhang, L.; Zhang, Y.; Gao, H.; Yan, H. Large-Area Perovskite Solar Cells - a Review of Recent Progress and Issues. RSC Adv. 2018, 8 (19), 10489-10508.

(2) Nie, W. Y.; Tsai, H. H.; Asadpour, R.; Blancon, J.-C. C.; Neukirch, A. J.; Gupta, G.; Crochet, J. J.; Chhowalla, M.; Tretiak, S.; Alam, M. A.; et al. High-Efficiency Solution-Processed Perovskite Solar Cells with Millimeter-Scale Grains. Science (Washington, DC, U. S.) 2015, 347 (6221), 522-525.

(3) Howard, I. A.; Abzieher, T.; Hossain, I. M.; Eggers, H.; Schackmar, F.; Ternes, S.; Richards, B. S.; Lemmer, U.; Paetzold, U. W. Coated and Printed Perovskites for Photovoltaic Applications. Adv. Mater. 2019, 31, 1806702.

(4) Ren, Y.-K.; Shi, X.-Q.; Ding, X.-H.; Zhu, J.; Hayat, T.; Alsaedi, A.; Li, Z.-Q.; Xu, X.-X.; Yang, S.-F.; Dai, S.-Y. Facile Fabrication of Perovskite Layers with Large Grains through a Solvent Exchange Approach. Inorg. Chem. Front. 2018, 5 (2), 348-353.

(5) Whitaker, J. B.; Kim, D. H.; Larson, B. W.; Zhang, F.; Berry, J. J.; van Hest, M. F. A. M.; Zhu, K. Scalable Slot-Die Coating of High Performance Perovskite Solar Cells. Sustain. Energy Fuels 2018, 2 (11), 2442-2449.

(6) Jin, J.; Li, H.; Chen, C.; Zhang, B.; Xu, L.; Dong, B.; Song, H.; Dai, Q. Enhanced Performance of Perovskite Solar Cells with Zinc Chloride Additives. ACS Appl. Mater. Interfaces 2017, 9 (49), 4287542882.

(7) Saliba, M. Polyelemental, Multicomponent Perovskite Semiconductor Libraries through Combinatorial Screening. Adv. Energy Mater. 2019, 9 (25), 1803754.

(8) Huang, Y.; Wu, S.; Chen, R.; Fang, S.; Zhang, S.; Wang, G.; Chen, W. Efficient Methylamine-Containing Antisolvent Strategy to Fabricate High-Efficiency and Stable FA0.85Cs0.15Pb(Br0.15I2.85) Perovskite Solar Cells. ACS Appl. Mater. Interfaces 2019, 11 (20), 18415-18422.

(9) Klug, M. T.; Osherov, A.; Haghighirad, A. A.; Stranks, S. D.; Brown, P. R.; Bai, S.; Wang, J. T.-W.; Dang, X.; Bulovic, V.; Snaith, H. J.; et al. Tailoring Metal Halide Perovskites through Metal Substitution: Influence on Photovoltaic and Material Properties. Energy Environ. Sci. 2017, 10 (1), 236-246.

(10) Abdi-Jalebi, M.; Dar, M. I.; Sadhanala, A.; Senanayak, S. P.; Franckevičius, M.; Arora, N.; Hu, Y.; Nazeeruddin, M. K.; Zakeeruddin, S. M.; Grätzel, M.; et al. Impact of Monovalent Cation Halide Additives on the Structural and Optoelectronic Properties of CH3NH3PbI3 Perovskite. Adv. Energy Mater. 2016, 6 (10), 1502472.

(11) Abdi-Jalebi, M.; Andaji-Garmaroudi, Z.; Cacovich, S.; Stavrakas, C.; Philippe, B.; Richter, J. M.; Alsari, M.; Booker, E. P.; Hutter, E. M.; Pearson, A. J.; et al. Maximizing and Stabilizing Luminescence from Halide Perovskites with Potassium Passivation. Nature 2018, 555, 497.

(12) Halder, A.; Chulliyil, R.; Subbiah, A. S.; Khan, T.; Chattoraj, S.; Chowdhury, A.; Sarkar, S. K. Pseudohalide (SCN-)-Doped MAPbI3 Perovskites: A Few Surprises. J. Phys. Chem. Lett. 2015, 6 (17), 34833489.

(13) Pazos-Outón, L. M.; Xiao, T. P.; Yablonovitch, E. Fundamental Efficiency Limit of Lead Iodide Perovskite Solar Cells. J. Phys. Chem. Lett. 2018, 9 (7), 1703-1711.

(14) Sarritzu, V.; Sestu, N.; Marongiu, D.; Chang, X.; Masi, S.; Rizzo, A.; Colella, S.; Quochi, F.; Saba, M.; Mura, A.; et al. Optical Determination of Shockley-Read-Hall and Interface Recombination Currents in Hybrid Perovskites. Sci. Rep. 2017, 7, 44629.

(15) Meggiolaro, D.; De Angelis, F. First-Principles Modeling of Defects in Lead Halide Perovskites: Best Practices and Open Issues. ACS Energy Lett. 2018, 3 (9), 2206-2222.

(16) Ball, J. M.; Petrozza, A. Defects in Perovskite-Halides and Their Effects in Solar Cells. Nat. Energy 2016, 1, 16149.

(17) Wang, J.; Fu, W.; Jariwala, S.; Sinha, I.; Jen, A. K.-Y.; Ginger, D. S. Reducing Surface Recombination Velocities at the Electrical 
Contacts Will Improve Perovskite Photovoltaics. ACS Energy Lett. 2019, 4 (1), 222-227.

(18) Sherkar, T. S.; Momblona, C.; Gil-Escrig, L.; Ávila, J.; Sessolo, M.; Bolink, H. J.; Koster, L. J. A. Recombination in Perovskite Solar Cells: Significance of Grain Boundaries, Interface Traps, and Defect Ions. ACS Energy Lett. 2017, 2 (5), 1214-1222.

(19) Fassl, P.; Zakharko, Y.; Falk, L. M.; Goetz, K. P.; Paulus, F.; Taylor, A. D.; Zaumseil, J.; Vaynzof, Y. Effect of Density of Surface Defects on Photoluminescence Properties in $\mathrm{MAPbI}_{3}$ Perovskite Films. J. Mater. Chem. C 2019, 7, 5285.

(20) Uratani, H.; Yamashita, K. Charge Carrier Trapping at Surface Defects of Perovskite Solar Cell Absorbers: A First-Principles Study. J. Phys. Chem. Lett. 2017, 8 (4), 742-746.

(21) Zhu, L.; Xu, Y.; Zhang, P.; Shi, J.; Zhao, Y.; Zhang, H.; Wu, J.; Luo, Y.; Li, D.; Meng, Q. Investigation on the Role of Lewis Bases in the Ripening Process of Perovskite Films for Highly Efficient Perovskite Solar Cells. J. Mater. Chem. A 2017, 5 (39), 20874-20881.

(22) Stavrakas, C.; Zhumekenov, A. A.; Brenes, R.; Abdi-Jalebi, M.; Bulović, V.; Bakr, O. M.; Barnard, E. S.; Stranks, S. D. Probing Buried Recombination Pathways in Perovskite Structures Using 3D Photoluminescence Tomography. Energy Environ. Sci. 2018, 11 (10), 28462852.

(23) Staub, F.; Hempel, H.; Hebig, J.-C.; Mock, J.; Paetzold, U. W.; Rau, U.; Unold, T.; Kirchartz, T. Beyond Bulk Lifetimes: Insights into Lead Halide Perovskite Films from Time-Resolved Photoluminescence. Phys. Rev. Appl. 2016, 6 (4), 44017.

(24) Lee, D. S.; Yun, J. S.; Kim, J.; Soufiani, A. M.; Chen, S.; Cho, Y.; Deng, X.; Seidel, J.; Lim, S.; Huang, S.; et al. Passivation of Grain Boundaries by Phenethylammonium in Formamidinium-Methylammonium Lead Halide Perovskite Solar Cells. ACS Energy Lett. 2018, 3 (3), 647-654.

(25) Zhang, H.; Wu, Y.; Shen, C.; Li, E.; Yan, C.; Zhang, W.; Tian, H.; Han, L.; Zhu, W.-H. Efficient and Stable Chemical Passivation on Perovskite Surface via Bidentate Anchoring. Adv. Energy Mater. 2019, 9, 1803573.

(26) Guo, Q.; Yuan, F.; Zhang, B.; Zhou, S.; Zhang, J.; Bai, Y.; Fan, L.; Hayat, T.; Alsaedi, A.; Tan, Z. Passivation of the Grain Boundaries of CH3NH3PbI3 Using Carbon Quantum Dots for Highly Efficient Perovskite Solar Cells with Excellent Environmental Stability. Nanoscale 2019, 11 (1), 115-124.

(27) Zhang, W.; Saliba, M.; Moore, D. T.; Pathak, S. K.; Hörantner, M. T.; Stergiopoulos, T.; Stranks, S. D.; Eperon, G. E.; AlexanderWebber, J. A.; Abate, A.; et al. Ultrasmooth Organic-inorganic Perovskite Thin-Film Formation and Crystallization for Efficient Planar Heterojunction Solar Cells. Nat. Commun. 2015, 6, 6142.

(28) Yang, Y.; Peng, H.; Liu, C.; Arain, Z.; Ding, Y.; Ma, S.; Liu, X.; Hayat, T.; Alsaedi, A.; Dai, S. Bi-Functional Additive Engineering for High-Performance Perovskite Solar Cells with Reduced Trap Density. J. Mater. Chem. A 2019, 7 (11), 6450-6458.

(29) Yang, M.; Zeng, Y.; Li, Z.; Kim, D. H.; Jiang, C.-S.; van de Lagemaat, J.; Zhu, K. Do Grain Boundaries Dominate Non-Radiative Recombination in CH3NH3PbI3 Perovskite Thin Films? Phys. Chem. Chem. Phys. 2017, 19 (7), 5043-5050.

(30) Gedamu, D.; Asuo, I. M.; Benetti, D.; Basti, M.; Ka, I.; Cloutier, S. G.; Rosei, F.; Nechache, R. Solvent-Antisolvent Ambient Processed Large Grain Size Perovskite Thin Films for High-Performance Solar Cells. Sci. Rep. 2018, 8 (1), 12885.

(31) Foley, B. J.; Girard, J.; Sorenson, B. A.; Chen, A. Z.; Scott Niezgoda, J.; Alpert, M. R.; Harper, A. F.; Smilgies, D.-M.; Clancy, P.; Saidi, W. A.; et al. Controlling Nucleation, Growth, and Orientation of Metal Halide Perovskite Thin Films with Rationally Selected Additives. J. Mater. Chem. A 2017, 5 (1), 113-123.

(32) Rong, Y.; Hou, X.; Hu, Y.; Mei, A.; Liu, L.; Wang, P.; Han, H. Synergy of Ammonium Chloride and Moisture on Perovskite Crystallization for Efficient Printable Mesoscopic Solar Cells. Nat. Commun. 2017, 8, 14555.

(33) Sanchez, S.; Hua, X.; Phung, N.; Steiner, U.; Abate, A. Flash Infrared Annealing for Antisolvent-Free Highly Efficient Perovskite Solar Cells. Adv. Energy Mater. 2018, 8 (12), 1702915.
(34) Sánchez, S.; Vallés-Pelarda, M.; Alberola-Borràs, J.-A.; Vidal, R.; Jerónimo-Rendón, J. J.; Saliba, M.; Boix, P. P.; Mora-Seró, I. Flash Infrared Annealing as a Cost-Effective and Low Environmental Impact Processing Method for Planar Perovskite Solar Cells. Mater. Today 2019, DOI: 10.1016/j.mattod.2019.04.021.

(35) Han, L.; Cong, S.; Yang, H.; Lou, Y.; Wang, H.; Huang, J.; Zhu, J.; Wu, Y.; Chen, Q.; Zhang, B.; et al. Environmental-Friendly Urea Additive Induced Large Perovskite Grains for High Performance Inverted Solar Cells. Sol. RRL 2018, 2 (7), 1800054.

(36) Feng, J.; Zhu, X.; Yang, Z.; Zhang, X.; Niu, J.; Wang, Z.; Zuo, S.; Priya, S.; Liu, S. F.; Yang, D. Record Efficiency Stable Flexible Perovskite Solar Cell Using Effective Additive Assistant Strategy. Adv. Mater. 2018, 30 (35), 1801418.

(37) Nalwa, H. S. Handbook of Nanostructured Materials and Nanotechnology, five volume set; Elsevier Science, 1999.

(38) Zhou, Y.; Yang, M.; Vasiliev, A. L.; Garces, H. F.; Zhao, Y.; Wang, D.; Pang, S.; Zhu, K.; Padture, N. P. Growth Control of Compact $\mathrm{CH} 3 \mathrm{NH} 3 \mathrm{PbI} 3$ Thin Films via Enhanced Solid-State Precursor Reaction for Efficient Planar Perovskite Solar Cells. J. Mater. Chem. A 2015, 3 (17), 9249-9256.

(39) Mali, S. S.; Hong, C. K.; Inamdar, A. I.; Im, H.; Shim, S. E. Efficient Planar N-i-p Type Heterojunction Flexible Perovskite Solar Cells with Sputtered $\mathrm{TiO} 2$ Electron Transporting Layers. Nanoscale 2017, 9 (9), 3095-3104.

(40) Shtukenberg, A. G.; Punin, Y. O.; Gunn, E.; Kahr, B. Spherulites. Chem. Rev. 2012, 112 (3), 1805-1838.

(41) Marentette, J. M.; Brown, G. R. Polymer Spherulites: I. Birefringence and Morphology. J. Chem. Educ. 1993, 70 (6), 435.

(42) Fu, W.; Vaughan, J.; Gillespie, A. Effects of Inorganic Anions on the Morphology of Sodium Oxalate Crystallized from Highly Alkaline Solutions. Cryst. Growth Des. 2014, 14 (4), 1972-1980.

(43) Craciun, V.; Craciun, D.; Wang, X.; Anderson, T. J.; Singh, R. K. Transparent and Conducting Indium Tin Oxide Thin Films Grown by Pulsed Laser Deposition at Low Temperatures. J. Optoelectron. Adv. Mater. 2003, 5, 401-408.

(44) Zhao, J.; Deng, Y.; Wei, H.; Zheng, X.; Yu, Z.; Shao, Y.; Shield, J. E.; Huang, J. Strained Hybrid Perovskite Thin Films and Their Impact on the Intrinsic Stability of Perovskite Solar Cells. Sci. Adv. 2017, 3 (11), eaao5616.

(45) Ryde, L. Application of EBSD to Analysis of Microstructures in Commercial Steels. Mater. Sci. Technol. 2006, 22 (11), 1297-1306.

(46) Kamaya, M.; da Fonseca, J. Q.; Li, L. M.; Preuss, M. Local Plastic Strain Measurement by EBSD. Advances in Experimental Mechanics V; Applied Mechanics and Materials; Trans Tech Publications, 2007; Vol. 7, pp 173-179.

(47) Rohrer, G. S.; Li, J.; Lee, S.; Rollett, A. D.; Groeber, M.; Uchic, M. D. Deriving Grain Boundary Character Distributions and Relative Grain Boundary Energies from Three-Dimensional EBSD Data. Mater. Sci. Technol. 2010, 26 (6), 661-669.

(48) Adhyaksa, G. W. P.; Brittman, S.; Abolins, H.; Lof, A.; Li, X.; Keelor, J. D.; Luo, Y.; Duevski, T.; Heeren, R. M. A.; Ellis, S. R.; et al. Understanding Detrimental and Beneficial Grain Boundary Effects in Halide Perovskites. Adv. Mater. 2018, 30 (52), 1804792.

(49) Zhang, T.; Long, M.; Yan, K.; Zeng, X.; Zhou, F.; Chen, Z.; Wan, X.; Chen, K.; Liu, P.; Li, F.; et al. Facet-Dependent Property of Sequentially Deposited Perovskite Thin Films: Chemical Origin and Self-Annihilation. ACS Appl. Mater. Interfaces 2016, 8 (47), $32366-$ 32375.

(50) Leblebici, S. Y.; Leppert, L.; Li, Y.; Reyes-Lillo, S. E.; Wickenburg, S.; Wong, E.; Lee, J.; Melli, M.; Ziegler, D.; Angell, D. K.; et al. Facet-Dependent Photovoltaic Efficiency Variations in Single Grains of Hybrid Halide Perovskite. Nat. Energy 2016, 1, 16093.

(51) Moerman, D.; Eperon, G. E.; Precht, J. T.; Ginger, D. S. Correlating Photoluminescence Heterogeneity with Local Electronic Properties in Methylammonium Lead Tribromide Perovskite Thin Films. Chem. Mater. 2017, 29 (13), 5484-5492.

(52) de Quilettes, D. W.; Vorpahl, S. M.; Stranks, S. D.; Nagaoka, H.; Eperon, G. E.; Ziffer, M. E.; Snaith, H. J.; Ginger, D. S. Impact of 
Microstructure on Local Carrier Lifetime in Perovskite Solar Cells. Science (Washington, DC, U. S.) 2015, 348 (6235), 683-686.

(53) Castro-Méndez, A.-F.; Hidalgo, J.; Correa-Baena, J.-P. The Role of Grain Boundaries in Perovskite Solar Cells. Adv. Energy Mater. 2019, 1901489.

(54) Mohan, V.; Jain, P. K. Spectral Heterogeneity of Hybrid Lead Halide Perovskites Demystified by Spatially Resolved Emission. J. Phys. Chem. C 2017, 121 (35), 19392-19400.

(55) Richter, J. M.; Abdi-Jalebi, M.; Sadhanala, A.; Tabachnyk, M.; Rivett, J. P. H.; Pazos-Outón, L. M.; Gödel, K. C.; Price, M.; Deschler, F.; Friend, R. H. Enhancing Photoluminescence Yields in Lead Halide Perovskites by Photon Recycling and Light Out-Coupling. Nat. Commun. 2016, 7, 13941.

(56) Staub, F.; Kirchartz, T.; Bittkau, K.; Rau, U. Manipulating the Net Radiative Recombination Rate in Lead Halide Perovskite Films by Modification of Light Outcoupling. J. Phys. Chem. Lett. 2017, 8 (20), 5084-5090.

(57) Crothers, T. W.; Milot, R. L.; Patel, J. B.; Parrott, E. S.; Schlipf, J.; Muller-Buschbaum, P.; Johnston, M. B.; Herz, L. M. Photon Reabsorption Masks Intrinsic Bimolecular Charge-Carrier Recombination in CH3NH3PbI3 Perovskite. Nano Lett. 2017, 17 (9), 57825789.

(58) Diab, H.; Arnold, C.; Lédée, F.; Trippé-Allard, G.; Delport, G.; Vilar, C.; Bretenaker, F.; Barjon, J.; Lauret, J.-S.; Deleporte, E.; et al. Impact of Reabsorption on the Emission Spectra and Recombination Dynamics of Hybrid Perovskite Single Crystals. J. Phys. Chem. Lett. 2017, 8 (13), 2977-2983.

(59) Staub, F.; Kirchartz, T.; Bittkau, K.; Rau, U. Manipulating the Net Radiative Recombination Rate in Lead Halide Perovskite Films by Modification of Light Outcoupling. J. Phys. Chem. Lett. 2017, 8 (20), 5084-5090.

(60) Reid, O. G.; Yang, M.; Kopidakis, N.; Zhu, K.; Rumbles, G. Grain-Size-Limited Mobility in Methylammonium Lead Iodide Perovskite Thin Films. ACS Energy Lett. 2016, 1 (3), 561-565.

(61) Mooney, J.; Kambhampati, P. Get the Basics Right: Jacobian Conversion of Wavelength and Energy Scales for Quantitative Analysis of Emission Spectra. J. Phys. Chem. Lett. 2013, 4 (19), 3316-3318.

(62) Savenije, T. J.; Ferguson, A. J.; Kopidakis, N.; Rumbles, G. Revealing the Dynamics of Charge Carriers in Polymer:Fullerene Blends Using Photoinduced Time-Resolved Microwave Conductivity. J. Phys. Chem. C 2013, 117 (46), 24085-24103. 